\title{
Cosmology with X-ray Cluster Baryons
}

\author{
Eric V. Linder \\ Berkeley Lab, University of California, Berkeley, CA 94720 USA \\ E-mail: evlinder@lbl.gov
}

\begin{abstract}
X-ray cluster measurements interpreted with a universal baryon/gas mass fraction can theoretically serve as a cosmological distance probe. We examine issues of cosmological sensitivity for current (e.g. Chandra X-ray Observatory, XMMNewton) and next generation (e.g. Con-X, XEUS) observations, along with systematic uncertainties and biases. To give competitive next generation constraints on dark energy, we find that systematics will need to be controlled to better than $1 \%$ and any evolution in $f_{\text {gas }}$ (and other cluster gas properties) must be calibrated so the residual uncertainty is weaker than $(1+z)^{0.03}$.
\end{abstract}

PACS numbers: 95.36.+x, 98.80.-k, 98.65.Cw

\section{Introduction}

An increasing number of cosmological methods have been suggested to explore the cosmological model and the accelerating universe. This diversity offers hope in understanding the nature of our universe, if the probes are robust and clear in interpretation. Astrophysical systematic uncertainties of a technique and its observations limit the cosmological leverage, despite statistical precision. Here we examine the level of control of systematics required for accurate cosmology estimation from the X-ray cluster distance method, sometimes called the baryon or gas mass fraction method, that assumes a universal baryon/gas mass fraction.

X-ray cluster observations of fewer than 50 clusters by the Chandra X-ray Observatory [1] and XMM-Newton [2] have already been claimed to yield cosmological constraints [3, 4, 5, 6]. For the future, there are prospects of more and deeper clusters, for example from Constellation-X [7] and XEUS [8]. We investigate the possible constraints, their cosmological degeneracies, and complementarity with other probes, giving special attention to the effect of hypothetical systematic floors in the achievable accuracy. While the X-ray cluster baryon technique requires understanding the influence of cosmology and astrophysics on the ingredients of cosmic geometry, mass distributions, and hydrodynamical gas properties, it also has rich observational data.

A number of recent papers have raised issues concerning the central assumption of universality of the cluster gas mass fraction, from observational, simulation, and theory points of view, e.g. 9 , 10, 11, 12, 13, 14, 15). Given this uncertainty (which this 
paper does not try to resolve), it is useful to investigate how an overall uncertainty in the technique, arising from whatever source, affects the cosmological conclusions. We emphasize that this paper presents purely an investigation of cosmological sensitivity to the innate parameter degeneracies and end-of-pipeline level of systematic uncertainties; we do not make claims for the error contribution from specific measurements or what level of end systematics is achievable.

In 92 we examine the cosmological sensitivity of the constant gas mass fraction technique based on parameter degeneracies and survey depth. Biases in the cosmological results arising from possible systematics are calculated in \$3. A brief review of the gas mass fraction technique, noting assumptions and possible areas for systematic uncertainties, is presented in the Appendix, along with a comment on bias from nonGaussian errors in the translation to a distance measure.

\section{X-ray Cluster Gas as Distance Indicator}

The X-ray flux from a galaxy cluster is related to the cosmological parameters we seek to estimate by

$$
F_{X}=\tilde{A} d_{a}^{-3} f_{\text {gas }}^{2}=A d_{a}^{-3}\left(\Omega_{b} / \Omega_{m}\right)^{2},
$$

(see the Appendix for a review of the derivation) where $d_{a}$ is the angular diameter distance to the cluster, $f_{\text {gas }}$ is the cluster gas mass fraction and $\left(\Omega_{b} / \Omega_{m}\right)$ the universal baryon mass fraction, where $\Omega_{b}$ is the baryon density in units of the critical density and $\Omega_{m}$ the matter density in units of the critical density. The quantities $\tilde{A}, A$ should be independent of redshift and cosmology.

In practice, one assumes $f_{\text {gas }}$ should be constant (basically the universal value) and interprets any deviation from constancy as a deviation from the fiducial cosmology, adjusting the cosmology until $f_{\text {gas }}(z)$ appears constant. However, if $f_{\text {gas }}$ is not truly constant over the redshift range of the survey then one will be led to an improper, biased cosmology. Several papers recently [9, 10, 11, 12, 13, 14, 15] indicate that the question of constancy is still open. Without weighing in on this controversy, or the astrophysical assumptions and resulting individual systematic uncertainties that go into deriving Eq. (1) (see the Appendix for a quick review), let us look at the big picture and ask at what level we must control the overall systematic uncertainties embodied in the proportionality factor $A$ (which includes both $f_{\text {gas }}$ and other possible uncertainties). Again, any redshift dependence of $A$ will bias the cosmology.

First, we investigate the cosmological parameter sensitivities and degeneracies. As the central cosmological quantity, we take the combination $X \equiv d_{a}^{-3}\left(\Omega_{b} / \Omega_{m}\right)^{2}$ from Eq. (11). In this section we assume that the baryon density is known, an optimistic assumption since Big Bang nucleosynthesis and cosmic microwave background measurements determine the quantity $\Omega_{b} h^{2}$ rather than $\Omega_{b}$, where $h$ is the reduced Hubble constant. In $\oint 3$ we return to $\Omega_{b}$ and other neglected "constants" of proportionality. In the Appendix we discuss cautions regarding cosmological analysis by transforming $X$ directly to a distance quantity or $f_{\text {gas }}$. 
Considering measurements $X(z)$ at various redshifts $z$, we calculate the cosmological sensitivity $\partial X / \partial p$ and joint likelihood contours by Fisher analysis (approximating the likelihoods as Gaussian) for the parameter set $p=\left\{\Omega_{m}, w_{0}, w_{a}\right\}$, with a fiducial flat $\Lambda C D M, \Omega_{m}=0.28$ model. The dark energy equation of state is modeled by $w(z)=w_{0}+w_{a}(1-a)$, where $a=1 /(1+z)$.

Figure 1 shows the run of unmarginalized cosmological parameter sensitivity with redshift. Clearly the matter density $\Omega_{m}$ is the best determined parameter, and furthermore its sensitivity $\partial X / \partial \Omega_{m}$ has substantial redshift dependence, so cluster measurements over a wide redshift range will likely improve constraints on $\Omega_{m}$. Sensitivity to dark energy parameters is much reduced, in particular to the time variation $w_{a}$. Note that at $z>1$, the sensitivity curves of $w_{0}$ and $w_{a}$ are not parallel so a deeper survey can break the degeneracy between them (though of course precision observations of high redshift X-ray clusters are more difficult).

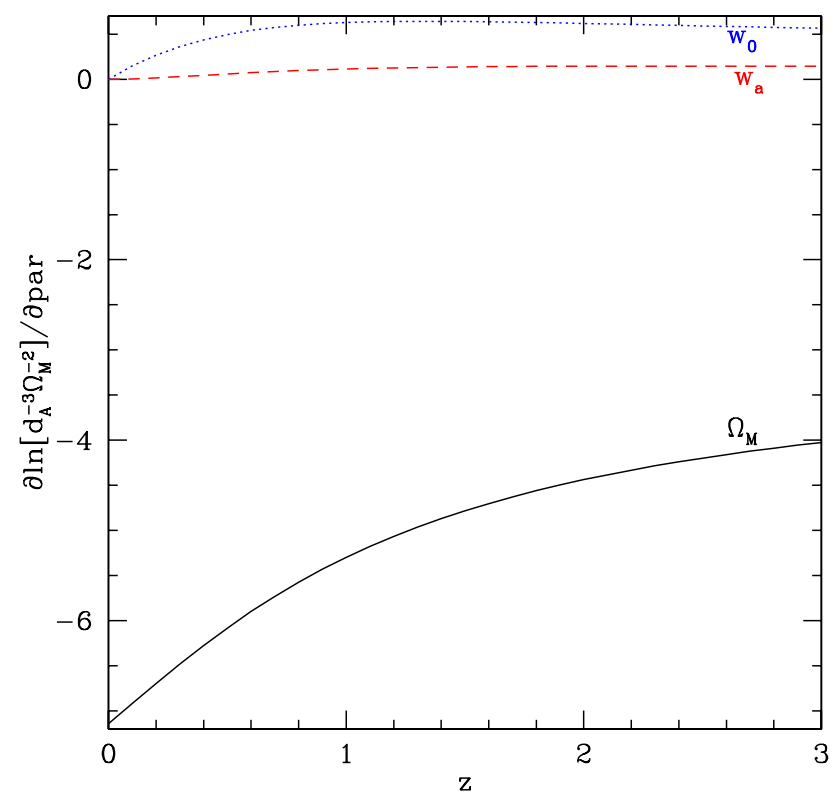

Figure 1. The sensitivity of the X-ray cluster observable $X \sim d_{a}^{-3} \Omega_{m}^{-2}$ to the cosmological parameters $p=\left\{\Omega_{m}, w_{0}, w_{a}\right\}$ for a flat universe are encoded in the derivatives plotted here. The larger the absolute magnitude of the derivative at a particular redshift, the more constraining the observations there.

To take into account the degeneracies between cosmological parameters, we employ a Fisher analysis involving $10 \%$ determinations of $X$ in each bin of 0.1 in redshift over the range $z=0.1-0.9$ or $z=0.1-1.7$. One can think of such precision naively as corresponding to better than $3.3 \%$ measurements of distance; we leave until $₫ 3$ the issue of what is the contribution of statistics vs. systematics to this number. This $10 \%$ value is purely illustrative; we consider later in this section what level of accuracy would be required to achieve substantial constraints on the dark energy parameters; in the absence 
of priors, errors and likelihood contours scale with the value adopted for the uncertainty. We also emphasize that these uncertainties are not measurement errors; they represent the amalgamated uncertainties (systematic plus statistical, with systematics likely to dominate) on the quantities entering the cosmological expression as defined by Eq. (1).

First, we look at the constraints on matter density and cosmological constant in a possibly nonflat universe. The contours in Fig. 2 (holding fixed $w=-1$ ) are strongly aligned to give tight constraints on $\Omega_{m}$, with much weaker bounds on $\Omega_{\Lambda}$. Indeed, $\Omega_{m}$ is 10-15 times better determined than $\Omega_{\Lambda}$. The orientation of the contour is in good agreement with similar plots in [3, 4, 6]. The extent of the contours will vary with different inputs for the redshift range and systematics level. Note we have also held the amplitude $A$ fixed (including the depletion bias and the baryon-gas offset, as well as fixing $\Omega_{b}$, all to be discussed in \$3), making this overidealized. Even so, a systematics level of $<2.5 \%$ (roughly equivalent to $0.8 \%$ distance measurements) is required to determine $\Omega_{\Lambda}$ to $5 \%$ from a survey to $z_{\max }=1.7$.

Using X-ray cluster gas mass as a probe of dark energy cosmology, where the equation of state is fit by $w_{0}$ and $w_{a}$ but restricting to a flat universe, we find that the constraints are less promising. If we restrict to a (physically unjustified) constant equation of state, then the X-ray data in the idealized case is oriented in basically the same direction as the CMB or weak lensing probes, with good orthogonality with supernova distance data. Allowing for the possibility of time variation of the dark energy equation of state, however, the insensitivity we saw in Fig. 1 means that the Xray technique is poor at uncovering the nature of dark energy. For cluster data extending out to $z=1.7$, even in the idealized case of fixed amplitude $A$, the constraints on dark energy are still $\sigma\left(w_{0}\right) \gtrsim 0.5$ and $\sigma\left(w_{a}\right) \gtrsim 2$; see Figure 3. (If one insists on considering a constant equation of state, the uncertainties can be found by taking a cut across the confidence contours, holding fixed $w_{a}=0$.)

Combining X-ray cluster gas mass measurements with other probes of dark energy does not greatly improve the constraints. X-ray data to $z_{\max }=1.7(\mathrm{XR})$ plus Planck $\mathrm{CMB}$ data on the distance to the last scattering surface still do not constrain $w_{0}$ to better than 0.4 or $w_{a}$ to better than 1. Adding XR to a baseline of supernovae (SN) to $z=1.7$ plus CMB plus weak lensing shear power spectrum (WL) of a $1000 \mathrm{deg}^{2} \mathrm{space}$ survey, tightens the area of the $w_{0}-w_{a}$ contour by only $2 \%$. Substituting XR instead of SN blows up the area by a factor 3. Furthermore, such a combination would possess no purely geometric probe of cosmology (independent of nonlinear structure formation and gas hydrodynamics).

To ensure that these conclusions are robust, we have carried out several further investigations. We have checked that the exact distribution of clusters within a given redshift range does not significantly affect the results. This is the case for other distance probes as well, where the optimum redshift distribution gives errors within a few percent of a flat distribution or a smoothly sculpted one [16]. For example, changing the uniform distribution to a Gaussian centered on $z_{\max } / 2$ with standard deviation $z_{\max } / 4$, our cosmological parameter estimations change by less than $4 \%(10 \%)$ for $z_{\max }=0.9(1.7)$. 

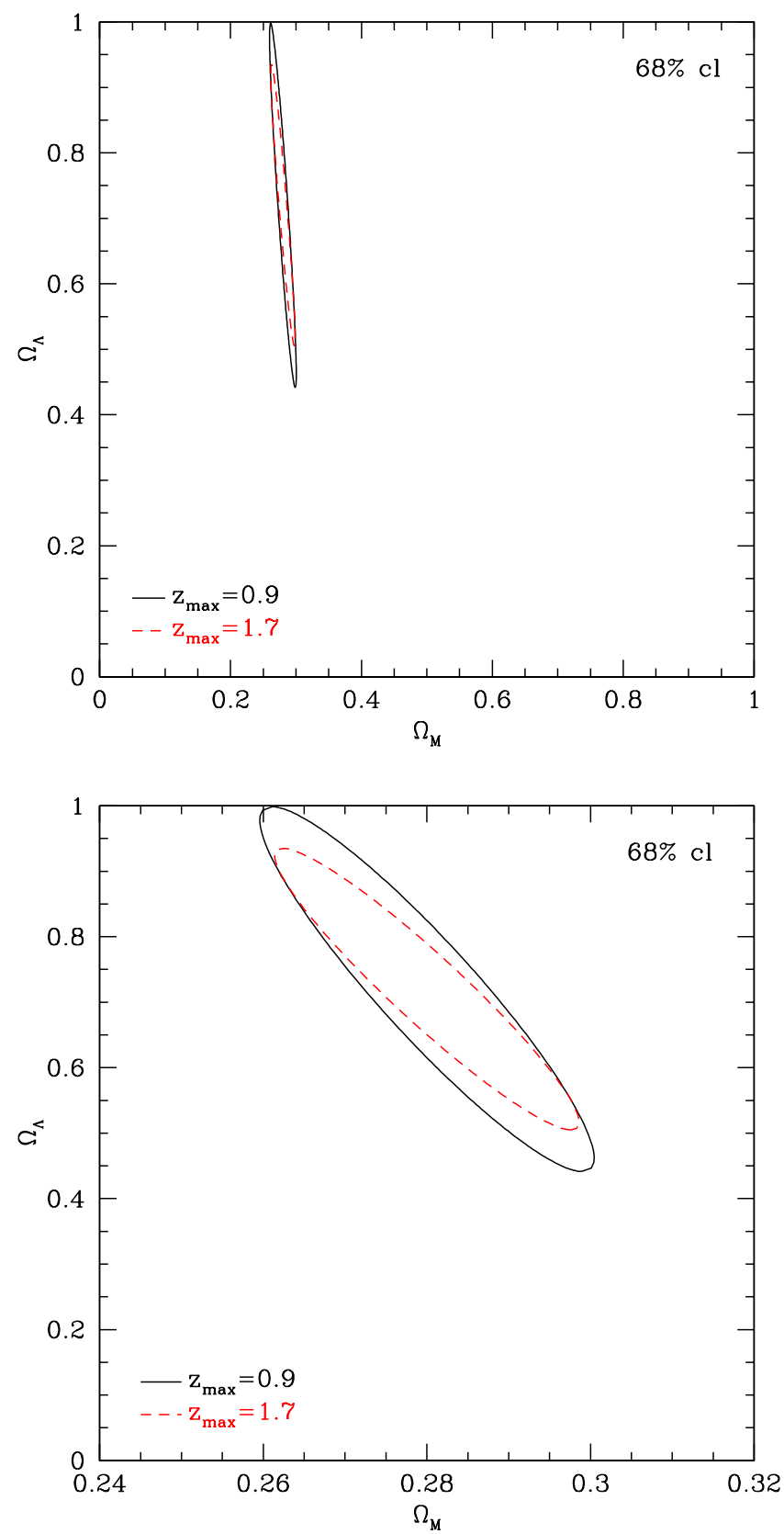

Figure 2. X-ray data constraints in the matter density vs. cosmological constant energy density plane act primarily to determine the matter density. The $1 \sigma$ joint confidence contours show the results from simulated X-ray cluster measurements out to $z=0.9$ (e.g. current Chandra observations) or $z=1.7$ (e.g. next generation Con-X observations), with $10 \%$ determinations of the parameter combination $X=d_{a}^{-3} \Omega_{m}^{-2}$ in each redshift bin of width 0.1 (under the idealized case of no systematic biases and other parameters fixed). The bottom panel zooms in on a region of the top panel.

From the sensitivity plot, Fig. 1, we had conjectured that extending the redshift range beyond $z \approx 1$ would continue to provide improved constraints (even in the $\Lambda$ 


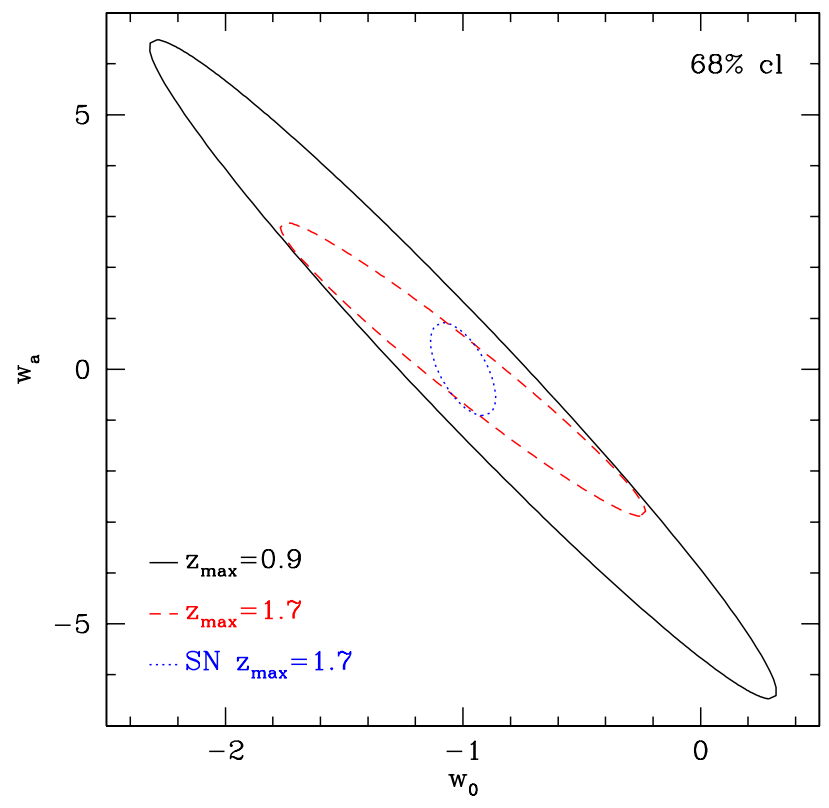

Figure 3. X-ray data constraints, simulated as in Fig. 2 (i.e. with idealized assumptions), provide only weak limits on the dark energy equation of state properties. Even a $z_{\max }=1.7 \mathrm{X}$-ray survey (e.g. Con-X depth) does not reveal dark energy properties at a level near the constraints expected from supernova distance measurements out to $z=1.7$ (e.g. SNAP quality, including systematics).

fiducial model). By keeping the same number of independent data points as for the $z_{\max }=1.7$ survey, but varying the survey depth $z_{\max }$, we confirm that the depth, not the greater number of statistical data bins as the redshift range increases, is predominantly responsible for improved cosmology estimation. The gain from depth saturates around $z_{\max } \approx 1.7$, e.g. the case with $z_{\max }=2$ only gives a further $5 \%(9 \%)$ improvement on $w_{0}\left(w_{a}\right)$.

If we think that we can neglect all systematics and continue to improve constraints purely with more statistics without a floor, then to achieve estimation of $w_{0}$ to better than 0.1 or $w_{a}$ to better than 0.5 , we require measurements of $X$ to $2 \%$ (roughly $0.67 \%$ distance accuracy) - although this still relies on an idealized analysis, without the necessary marginalizations and possible biases discussed in the next section. This appears to be challenging for the required level of astrophysical understanding and telescope time ([6] estimates that applying this technique to $250-500$ clusters will require $10 \%$ of all Con-X observing time in the first five years).

\section{Systematics and Biases}

The analysis up to this point of X-ray data has been idealized by ignoring possible systematic effects from the non-constancy of $A$ (including $f_{\text {gas }}$ ) in Eq. (1). That is, even if the precision of a sample at a given redshift is extremely good, a trend with redshift 
acts as a bias on cosmological parameter estimation. Therefore, any uncertainty on such a trend acts as a systematic uncertainty of the results.

As mentioned in the Appendix, one possible uncertainty concerns the recognized correction of the gas mass fraction by the additional factor [4]

$$
f_{\text {gas }} \sim[b /(1+0.19 \sqrt{h})] f_{\text {gas }}^{\text {ideal }},
$$

representing the effects of depletion bias $b$ of baryons leaking out of clusters during formation and relaxation [17], and the correction for cluster baryons present in galaxies rather than intracluster gas [18]. Recall that $f_{\text {gas }}$ enters squared into $X$. This, and other effects outlined in the Appendix that characterize the gas properties, can all be examined through the amplitude factor $A$. If this parameter is not assumed a priori fixed, it must be marginalized over (similar to the case of the absolute magnitude in supernova cosmology); since we have ignored this up to now, cosmological constraints from X-ray data derived in the previous section are overoptimistic. The uncertainties in $A$, as well as possible drifts with redshift in its value, e.g. from evolving cluster or cluster environment properties, generate systematic floors beyond which statistical improvement is cut off.

Taking proper account of the marginalization over $A$ (which in principle includes not only the astrophysical factors, but the baryon density $\Omega_{b}$ ) will act to blow up the uncertainties on the cosmological parameters, especially $\Omega_{m}$ and $w_{a}$. If $A$ is not limited a priori, then to achieve cosmology estimation with $\sigma\left(w_{a}\right)<1$ we find measurement of thousands of times as many clusters are required than when $A$ is assumed completely known. For this many clusters to contribute usefully, systematic uncertainties must be bounded below the $0.2 \%$ level.

First consider the case where $A$ is a random variable, independent of redshift. If we understand the gas hydrodynamics and other astrophysical uncertainties, using the full range of X-ray and other cluster measurements, we can constrain $A$ to some precision with a prior. In the previous section we found that in order to obtain significant constraints on dark energy, e.g. knowing $w_{a}$ to within 0.5 , we required a systematics level of less than $2.6 \%$. Given that level, but now removing the idealization of perfectly known $A$, calculations indicate that predicting $A$ to $3 \%(10 \%)$ degrades the estimation of $\Omega_{m}$ by $30 \%$ (a factor 3 ).

Now consider not a statistical uncertainty, but a systematic trend in redshift. Again, the array of precise X-ray measurements can attempt to correct for this by fitting the evolution in $A$ with extra parameters - if we know the functional form (sometimes called self-calibration). Suppose we guess $A=A_{0}(1+z)^{\alpha}$. Even with $A_{0}$ fixed, fitting for $\alpha$ blows up beyond use the cosmological parameter determination, i.e. pure selfcalibration fails. We therefore require priors for $A_{0}$ and $\alpha$, i.e. we must understand the astrophysical properties of clusters sufficiently well to limit these parameters. We find that $A_{0}$ basically is degenerate with $\Omega_{m}$ (cf. Eq. 1) and $\alpha$ has strong degeneracy with $w_{a}$.

If we misestimate the values of the calibration parameters then the residual 
systematics bias the cosmology. For example, mistaking $A_{0}$ leads to a bias $\delta \Omega_{m} / \sigma\left(\Omega_{m}\right)=$ $0.73\left(\delta A_{0} / 0.1\right)$. If we require keeping the offset below $0.46 \sigma$ (so the risk, the square root of the quadratic sum of the statistical and systematic errors, is degraded by less than $10 \%$ ), then $A_{0}$ must be known to 0.06. Such a redshift independent shift (as opposed to the previous dispersion) does not have a strong effect on the cosmological model.

Residual uncertainty in the redshift dependence, in the simplest case the value of the power law index $\alpha$, is more significant. Perhaps the simplest scenario is assuming, say, the depletion factor $b$ in Eq. (2) is universal, when in fact it has some evolution $\sim(1+z)^{c}$ (hence $\alpha=2 c$ ). Figure 4 shows the rather dramatic results of bias in the cosmological parameters generated by an evolution as slow as $c= \pm 0.25$. (We plot $1 \sigma$ projected, or $39 \%$ confidence level, contours so the biases can read off directly from projection to the axes.) Such misestimated evolution can bias the true cosmology by $3 \sigma$.

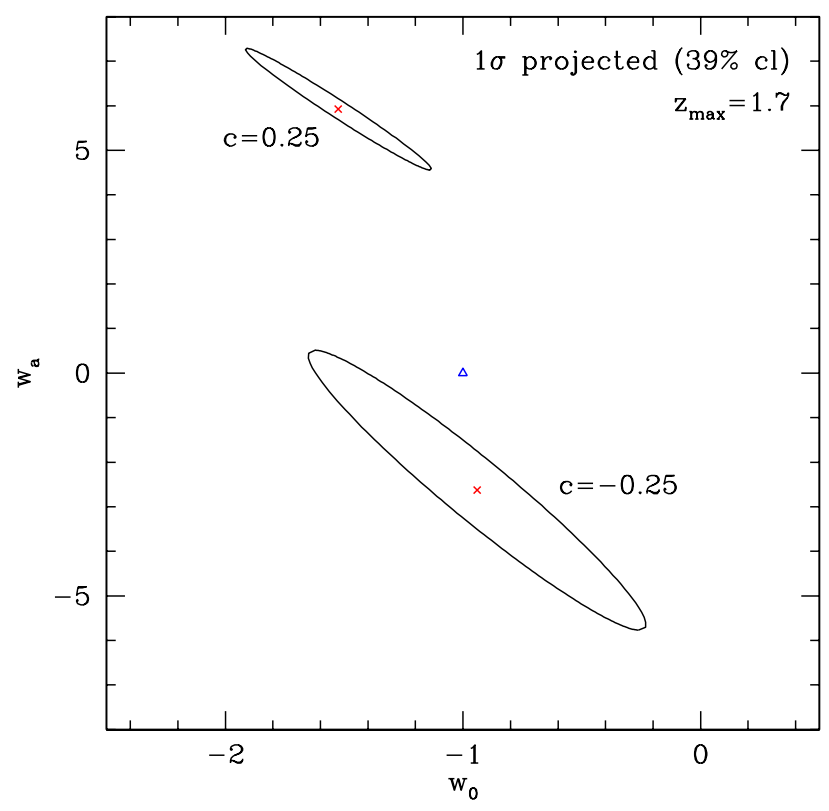

Figure 4. Systematic uncertainties in X-ray cluster properties can strongly bias the cosmological parameter estimation. A mild evolution in the baryon depletion or galaxy contribution factors (see Eq. 2), for example, going with redshift as $(1+z)^{c}$ can shift the derived cosmology (shown in each case by x's) by several standard deviations from the true cosmology (given by the open triangle).

To ensure $w_{a}$ is biased less than 0.5 off the true value, one requires $\delta c<0.03$. That is, an error in understanding redshift evolution of $f_{\text {gas }}$ of $(1+z)^{0.03}$ will invalidate the cosmology estimation. This is further complicated by the fact that measuring the baryon depletion $b$ from observations is itself entangled with cosmology. Knowing $c$ to 0.03 implies an understanding of baryons in clusters, at low and high redshift, such that a drift of $3 \%$ in $b$ from $z=0$ to 1.7 can be ruled out. Furthermore, gas properties 
discussed in the Appendix, such as clumpiness, and temperature and pressure profiles, multiply $f_{\text {gas }}$ in the amplitude factor $A$ and so the entire product must be subject to this tight systematics control. Note that self-calibration does not immunize against such systematic errors unless the adopted evolutionary function is exact - i.e. one must know the form of the variation beforehand for self-calibration to work.

In addition to physical evolution in cluster properties, systematic uncertainties can also arise from population drift, e.g. if there are multiple populations with different baryon depletion factors, say, and population $i$ is more prevalent at low redshift and population $j$ dominates at high redshift. We have seen that such redshift dependent systematics have a strong influence on $w_{a}$, while conversely the inherent sensitivity of the X-ray cluster baryon/gas mass fraction method to $w_{a}$ is low (see Fig. 1), so it appears quite challenging for even next generation X-ray cluster distances to provide robust constraints on dark energy properties.

To estimate in detail where the systematic floor lies for this technique is beyond the scope of this paper, and will require substantial understanding of cluster mass distributions and gas properties. Some of the possible systematics identified are listed in the Appendix. We have shown that in order to achieve determination $\sigma\left(w_{a}\right)<0.5$ we must limit the redshift independent systematic uncertainty to below $0.7 \%$ on both the gas mass fraction and the distance determination. If such a systematic floor could be reached, then matching this error level with statistics would require a sample of more than

$$
N=3400\left(\frac{D}{0.1}\right)^{2} \text { clusters }
$$

out to $z_{\max }=1.7$, to statistically reduce an individual cluster dispersion of $D$ down to a level $0.7 \%=D / \sqrt{N}$. For the combination of the X-ray technique with Planck CMB data, achieving $\sigma\left(w_{a}\right)<0.5$ requires $1 \%$ systematics and 1600 tightly characterized clusters. Note that proposed large programs for Con-X may measure a few hundred clusters [6].

As we saw, the redshift dependent systematic uncertainty has more severe effects. We must know not only the functional form of any evolution or population drift in cluster properties, but fit the parameters of the evolution to high accuracy. For example, a residual drift more rapid than $(1+z)^{0.03}$ will bias the time variation of the dark energy equation of state, $w_{a}$, by more than 0.5 .

\section{Conclusion}

We have considered the possibility of current and next generation observations of Xray clusters as cosmological distance probes through the baryon/gas mass fraction method. In current and future usage, assuming a cosmological constant universe, this technique can provide tight constraints on the matter density. However, even with the next generation Con-X and XEUS observatories, we find that it will be a considerable challenge for such X-ray cluster measurements to provide a precision dark energy probe. 
As with any probe, we cannot ignore possible systematic uncertainties. While many have been identified (see the Appendix for a brief discussion), the level of systematics control possible is currently not well determined. Understanding of cosmic geometry, the nonlinear mass distribution, and gas hydrodynamics are simultaneously required. The amplitude factor $A$ must be fit for, and redshift dependent uncertainties from evolution or population drift are especially degenerate with the dark energy equation of state time variation. Self-calibration only works if the functional form of the evolution is known and if the redshift scaling can be calculated to within $(1+z)^{0.03}$.

$\mathrm{X}$-ray cluster studies from simulations, theory, and observations over the next decade will address what is a realistic level of systematic uncertainties, both as a floor on precision at a given redshift and as a redshift dependent bias. We emphasize that this paper does not claim what level should be adopted but provides a general investigation of what level will impact cosmological parameter determination. Even if the X-ray gas mass fraction technique turns out not to be significant as a dark energy probe, the Xray data is likely to greatly extend and deepen our knowledge of cluster properties and evolution.

\section{Acknowledgments}

I thank Jochen Weller and especially the referee for helpful comments. This work has been supported in part by the Director, Office of Science, Department of Energy under grant DE-AC02-05CH11231.

\section{Appendix: Review of Method}

The gas mass fraction of a galaxy cluster, traced through X-ray measurements, is neither precisely a baryon fraction measurement nor a distance indicator. It is useful to briefly, if basically, review the quantities that enter, and under what assumptions. We follow the approach of [19]; also see [4, 5]. As with any cosmological probe, systematic controls are necessary for each assumption.

The measured X-ray flux $F_{X}$ is related to the intrinsic cluster X-ray luminosity $L_{X}$ and the cosmological luminosity distance $d_{l}$ to the cluster by

$$
F_{X}=\frac{L_{X}}{4 \pi d_{l}^{2}}
$$

The luminosity distance is $H_{0} d_{l}=(1+z)\left(1-\Omega_{T}\right)^{-1 / 2} \sinh \left[\left(1-\Omega_{T}\right)^{1 / 2} \int_{0}^{z} d z^{\prime} /\left[H\left(z^{\prime}\right) / H_{0}\right]\right]$, where $\Omega_{T}=\Omega_{m}+\Omega_{w}$ is the total density in units of the critical density, and the Hubble parameter $\left[H(z) / H_{0}\right]^{2}=\Omega_{m}(1+z)^{3}+\Omega_{w}(1+z)^{3\left(1+w_{0}+w_{a}\right)} e^{-3 w_{a} z /(1+z)}+(1-$ $\left.\Omega_{T}\right)(1+z)^{2}$. To use the cluster as a cosmological distance probe we need to understand the astrophysical and cosmological dependence of the cluster luminosity. This adds requirements on understanding "mass+gas", i.e. nonlinear structure formation and gas hydrodynamics, to the geometric distance. (See the cosmology probe classifications discussed in [20].) For an analogous situation of astrophysical dependence of luminosity 
when using Type Ia supernovae as distance probes, and methods of treatment, see, e.g., [21].

The X-ray luminosity (taking into account the main dependencies) is

$$
L_{X} \sim n_{e}^{2} T^{1 / 2} d_{a}^{3}
$$

where $n_{e}$ is the free electron density, $T$ is the gas temperature, and $d_{a}=d_{l} /(1+z)^{2}$ is the angular diameter distance to redshift $z$. There are several well known issues. The electron density enters squared, which makes cluster core detection easier but introduces sensitivity to substructure or clumpiness $\left\langle n_{e}^{2}\right\rangle /\left\langle n_{e}\right\rangle^{2}$. The gas is assumed to be at an equilibrium temperature. High resolution and spectral observations can test both of these to some extent. The distance enters through the volume the gas is occupying, given an observed angular size, which uses an assumption of spheroidal symmetry to deproject the two dimensional X-ray measurements to the three dimensional gas distribution. The distribution may be spatially complex, with filaments and voids, but again this can be checked to some extent through observations. If the cluster gas is relaxed to a smooth, ellipsoidal shape, one might think the average over orientations of many clusters would still give the correct luminosity. However, there is a bias in that clusters prolate along the line of sight have a higher projected flux and hence are more easily detected; this amplification is proportional to the quadrupole moment of the distribution. If the cluster flux is near the detection threshold then the quadrupole distribution does not average to $\langle Q\rangle=0$ but is offset due to selection bias.

The electron density at some radius $R$ is proportional to the gas mass within a shell at that radius, $d M_{g} \sim n_{e} R^{2} d R$. To relate the radius to an astrophysical property of the cluster requires an assumption such as hydrostatic equilibrium,

$$
G \mu m_{H} \frac{M_{t o t}(<R)}{R}=-k T\left(\frac{d \ln n_{e}}{d \ln R}+\frac{d \ln T}{d \ln R}\right),
$$

where $\mu m_{H}$ is the average mass per baryon. Under the further assumption that the right hand side is cosmology independent, i.e. depending only on the local cluster properties, we find that $M_{t o t} \sim R \sim d_{a}$. It is not clear that either of these assumptions is guaranteed. For example, if we choose $R$ to be at a fixed multiple of either the critical or background density at the cluster redshift, e.g. 2500 times $\left(r_{2500}\right)$, then the mass depends on the cosmology and matter density. This is the standard problem with defining the mass of a cluster, since that is not necessarily a unique quantity. Furthermore, even if the cluster profile is universal, the concentration (related to the overdensity relative to the scale radius [22]) depends on cosmology [23, affecting the right hand side of Eq. (A.3. As well, the temperature is possibly the greatest source of observational uncertainty and must be measured at the same radius as used for the cosmology; it will also be affected by non-uniformity or flux dependence of the spectral response of the X-ray instrumentation.

Carrying forward regardless, we obtain from translating $n_{e}$ into $M_{g}$ and $R$ into $M_{\text {tot }}$ 
that

$$
F_{X} \sim R^{-1} d_{a}^{-2}\left(\frac{d M_{g}}{d M_{t o t}}\right)^{2} \sim d_{a}^{-3}\left(\frac{d M_{g}}{d M_{t o t}}\right)^{2} \sim d_{a}^{-3}\left(\frac{\Omega_{b}}{\Omega_{m}}\right)^{2} .
$$

The last expression converts from gas quantities to baryon quantities. A major assumption in the use of Eq. (A.4) is that the gas mass fraction $M_{g} / M_{\text {tot }}$ measured for any cluster is equal to a constant quantity $f_{\text {gas }}$, and that furthermore this is the universal baryon to matter ratio $\Omega_{b} / \Omega_{m}$. In fact, since baryons are present in cluster galaxies as well as the intracluster (X-ray emitting) gas, and baryons are also lost during cluster formation, one must make a correction as in Eq. (2)).

We thus end up with the central cosmological dependence shown in Eq. (1), if the assumptions hold or can be corrected for. All the factors of non-universality of gas clumpiness, gas pressure and temperature profiles, and the gas mass fraction $f_{\text {gas }}$, multiply together to form the amplitude factor $A$ for the proportionality in Eq. (A.4). Any cosmology dependence in the gas parameters in $A$ alters the cosmology deduced for the true cosmological quantities of the distance $d_{a}$ and matter and baryon densities $\Omega_{m}$ and $\Omega_{b}$.

An additional point that may seem straightforward, but does have the potential to be overlooked, concerns a nonlinear transformation of the analysis variables. We use $X$, as the quantity most closely related to observables. If phrased in terms of $f_{\text {gas }}$ or $d_{a}$, the transformation is nonlinear, and Gaussian errors in the observables will become non-Gaussian. Put most simply, $\left\langle d_{a}\right\rangle \sim\left\langle F_{X}^{-1 / 3}\right\rangle \neq\left\langle F_{X}\right\rangle^{-1 / 3}$. As is known from the analysis of supernovae, when transforming fluxes into magnitudes (logarithms of flux), such non-Gaussianities, if overlooked, can lead to bias in the derived cosmology, as emphasized generally by [24]. This can be nontrivial: for example, a Gaussian dispersion $\sigma_{X} / X=0.02 z$ that is analyzed in terms of $f_{\text {gas }}$ with Gaussian errors will turn a true cosmological constant cosmology into one that looks like evolving dark energy with $w_{a} \approx-0.5$.

\section{References}

[1] Chandra X-ray Observatory, http://chandra.harvard.edu

[2] XMM-Newton, http://sci.esa.int/xmm

[3] Ettori S, Tozzi P and Rosati P 2003 Astron. Astrophys. 398879

[4] Allen S W et al. $2004 M N R A S 353457$

[5] Rapetti D, Allen S W and Weller J 2005 MNRAS 360555

[6] Rapetti D, Allen S W and Con-X Facility Science Team Preprint astro-ph/0608009

[7] Constellation-X Observatory, http://constellation.gsfc.nasa.gov

[8] X-ray Evolving Universe Spectrometer (XEUS), http://www.rssd.esa.int/XEUS

[9] Vikhlinin A et al. 2006 Ap. J. 640691

[10] Ettori S, Dolag K, Borgani S and Murante G 2006 MNRAS 3651021

[11] Hallman E J, Motl P M, Burns J O and Norman M L Preprint astro-ph/0509460

[12] Loeb A Preprint astro-ph/0606572

[13] Nagai D, Vikhlinin A and Kravtsov A V Preprint astro-ph/0609247

[14] Ferramacho L D and Blanchard A Preprint astro-ph/0609822

[15] Gastaldello F et al., Preprint astro-ph/0610134 
[16] Huterer D and Turner M S 2001 Phys. Rev. D 64123527

[17] Eke V R, Navarro J F and Frenk C S 1998 Ap. J. 503569

[18] White S D M, Navarro J F, Evrard A E and Frenk C S 1993 Nature 366429

[19] Sasaki S 1996 Publ. Astron. Soc. Japan 48 L119

[20] Linder E V 2005 in DARK2004, Proc. 5th Intl. Heidelberg Conf. (Berlin: Springer-Verlag) p 485 (Preprint astro-ph/0501057)

[21] Branch D, Perlmutter S, Baron E and Nugent P 2001, in Resource Book on Dark Energy (Preprint astro-ph/0109070)

[22] Navarro J F, Frenk C S and White S D M 1997 Ap. J. 490493

[23] Bartelmann M et al. 2005 New Astron. Rev. 49199

[24] Holz D E and Linder E V 2005 Ap. J. 631678 